\title{
What Works in the Treatment of Borderline Personality Disorder
}

\author{
Lois W. Choi-Kain ${ }^{1}$ • Ellen F. Finch ${ }^{1} \cdot$ Sara R. Masland ${ }^{1}$. James A. Jenkins ${ }^{2}$ • \\ Brandon T. Unruh ${ }^{1}$
}

Published online: 3 February 2017

(C) The Author(s) 2017. This article is published with open access at Springerlink.com

\begin{abstract}
Purpose of the Review This review summarizes advances in treatments for adults with borderline personality disorder (BPD) in the last 5 years.

Recent Findings Evidence-based advances in the treatment of BPD include a delineation of generalist models of care in contrast to specialist treatments, identification of essential effective elements of dialectical behavioral therapy (DBT), and the adaptation of DBT treatment to manage post-traumatic stress disorder (PTSD) and BPD. Studies on pharmacological interventions remain limited and have not provided evidence that any specific medications can provide stand-alone treatment.

Summary The research on treatment in BPD is leading to a distillation of intensive packages of treatment to be more broadly and practically implemented in most treatment environments through generalist care models and pared down forms of intensive treatments (e.g., informed case management plus DBT skills training groups). Evidence-based integrations of DBT and exposure therapy for PTSD provide support for changing practices to simultaneously treat PTSD and BPD.
\end{abstract}

This article is part of the Topical Collection on Personality and Impulse Control Disorders

Lois W. Choi-Kain

1choikain@partners.org

1 Harvard Medical School, McLean Hospital, 115 Mill St., Mail Stop 312, Belmont, MA 02478, USA

2 Massachusetts General Hospital, McLean Hospital, Belmont, MA 02478, USA
Keywords Borderline personality disorder · Psychotherapy · General psychiatric management $\cdot$ Dialectical behavior therapy $\cdot$ Mentalization-based treatment .

Transference-focused psychotherapy $\cdot$ Schema-focused therapy

\section{Introduction}

Once thought to be an untreatable condition, borderline personality disorder (BPD) is now effectively treated by a growing number of evidence based psychotherapeutic treatments. Twenty-five years ago, Marsha Linehan published the first randomized control trial (RCT) for dialectical behavioral therapy (DBT), which yielded more significant reduction in the disorder's most challenging features - parasuicidal behavior, inpatient psychiatric stays, and treatment drop out - than treatment as usual (TAU). Since then, over 13 manualized psychotherapies for BPD have been tested. Five major treatmentsDBT, mentalization-based treatment (MBT) [1], schemafocused therapy (SFT) [2], transference-focused psychotherapy (TFP) [3], and systems training for emotional predictability and problem solving (STEPPS) [4] - have been established as evidence based treatments (EBTs) for BPD [5]. In contrast, trials testing psychopharmacologic treatments have not yielded consistent results [6, 7]; therefore, to date no medication has an official indication for BPD [8•]. This review will summarize major findings of what works in treatments for BPD, with a special emphasis on developments in the last 5 years.

The Cochrane review of psychological therapies for borderline personality disorder, which analyzed 28 studies published until 2011, is among the most significant additions to the literature on treatments for BPD in the last 5 years [5]. The major randomized controlled studies can be characterized in 
Table 1 Major waves of RCTs for BPD treatment

\begin{tabular}{|c|c|c|}
\hline Wave 1: vs. TAU & & \\
\hline DBT & Linehan et al. [11] & 1991 \\
\hline DBT & Linehan et al. [10] & 1994 \\
\hline DBT & Koons et al. [9] & 2001 \\
\hline DBT & Carter et al. [12] & 2010 \\
\hline DBT & Priebe et al. [13] & 2012 \\
\hline DBT PTSD & Steil et al. [14] & 2010 \\
\hline DBT PTSD & Bohus et al. [15•] & 2013 \\
\hline MBT & Bateman \& Fonagy [1] & 1999 \\
\hline MBT-A & Rossouw \& Fonagy [16] & 2012 \\
\hline $\mathrm{CBT}+\mathrm{TAU}$ & Davidson et al. [17] & 2006 \\
\hline MACT & Weinberg et al. [18] & 2006 \\
\hline STEPPS + TAU & Blum et al. [4] & 2008 \\
\hline STEPPS + adjunctive therapy & Bos et al. [19] & 2010 \\
\hline DDP & Gregory et al. [20] & 2008 \\
\hline Wave 2: vs. community experts & & \\
\hline DBT & Linehan et al. [21] & 2006 \\
\hline TFP & Doering et al. [22] & 2010 \\
\hline Wave 3: vs manualized treatment & & \\
\hline SFT vs TFP & Giesen-Bloo et al. [2] & 2006 \\
\hline DBT vs DDP vs TAU & Sachdeva et al. [23] & 2013 \\
\hline TFP vs DBT vs supportive therapy & Clarkin et al. [3] & 2007 \\
\hline Wave 4: vs. generalized treatment & & \\
\hline DBT vs GPM & McMain et al. [24] & 2009 \\
\hline MBT vs SCM & Bateman \& Fonagy [25] & 2009 \\
\hline MBT vs supportive group therapy & Jørgensen et al. [26] & 2013 \\
\hline
\end{tabular}

four major waves (Table 1). The first wave of studies compared specialized therapies for BPD to TAU. In this first wave of studies, DBT and MBT were established as EBTs [1, 9-11]. Additionally, a short-term group therapy, STEPPS, was added to TAU and found to be more effective than TAU alone in reducing symptoms of BPD, negative mood states, and impulsivity while increasing functioning [4].

Responding to criticisms that treatments lead by experts had an obvious advantage to TAU, investigators in subsequent trials in the second wave compared specialized BPD treatments, such as DBT and TFP, to treatment by expert therapists in the community, known for their willingness and interest in patients with BPD [21, 22]. DBT again showed higher reduction in suicidal behavior and self-injury, inpatient hospitalization, and treatment drop out than treatment by other experts in the community [21]. Similarly, in the trial comparing TFP to treatment by community experts, TFP yielded greater improvements in not only suicide attempts, inpatient hospitalization, and treatment drop out, but also in borderline symptoms, psychosocial functioning, and personality functioning [22]. Treatment by experts was as effective as specialty treatments in reducing depressive symptoms, and in the DBT trial, treatment by experts was effective in reducing suicidality, but not to the degree achieved in DBT [21,22].

The third wave of studies staged head-to-head trials between specialist treatments (i.e., TFP vs. DBT [3]; SFT vs. TFP [2]). The evidence suggested a variety of systematic and well-informed manualized psychotherapies were effective at treating BPD and little was to be gained at horseracing to determine the superiority of any of them [27]. Clarkin's TFP trial straddles both the third and fourth wave of studies [3]. It incorporated a systematic, manualized supportive therapy as a third comparison treatment to TFP and DBT. All three treatments, which were systematic and supervised, yielded improvements in depression, anxiety, and functioning [3]. While the supportive therapy arm failed to match reductions in suicidality yielded by both DBT and TFP, it proved effective enough to be an alternative to treating BPD patients in the absence of DBT and TFP treatments.

The other fourth wave of studies compared specialist therapies to systematic and well-informed generalist approaches to managing BPD. These studies aimed to show that the core ingredients to the specialist treatments provided increased gains in treatment, apart from the well organized and wellinformed aspects of the clinical approach [3]. Unexpectedly, these enhanced, structured, and well-informed generalist treatment approaches performed as well in most ways to their already established specialized counterparts. Structured clinical management (SCM) $[25,28]$ and general psychiatric management (GPM) $[8 \cdot, 24]$ emerged to provide less specialized means of managing BPD in the general patient population. Additionally, supportive therapy, when delivered in a systematic way [3] or by experienced clinicians in a group format [26], proved comparable in outcomes to TFP and MBT. When clinicians treating a general caseload of patients cannot often commit the time and expense to training for the gold standard specialist treatments for BPD [29], SCM, GPM, and supportive therapy offer practical, less intensive, "good enough" treatment options.

While generalist approaches to BPD could widen access to evidence-based care, research dismantling differing aspects of effective, but complex and intensive treatments, can clarify their essential elements. Linehan and colleagues published a dismantling study of DBT, illustrating that a simplified version of DBT, involving skills training group combined with weekly case management was effective in treating problems of self-harm, suicidality, and use of hospitalization [30•]. Generalist care, with the addition of targeted short-term adjunctive interventions in group formats or aimed at suicidality $[4,18,31]$ may complement supportive or generalist approaches to yield good outcomes, with the investment of fewer clinical resources.

Investigators have also adapted the established evidence based treatments for BPD to manage the usual complex comorbidities of BPD including substance use disorders 
substance use disorders (SUDs) [32], eating disorders (EDs) [33], and post-traumatic stress disorder (PTSD) [34]. BPD patients who present with acutely symptomatic comorbidities of these types are often challenging to manage with strictly BPD oriented treatments [35]. Conversely, in SUD and ED treatments, individuals with co-morbid BPD may also present with problems that are difficult to manage in those treatment environments. Efforts to target BPD with its co-morbid disorders simultaneously have been developed and studies of their feasibility and effectiveness have been published in the last 5 years $[15 \cdot, 32,33,36,37,38]$.

The remainder of this review will describe the prevailing evidence-based psychotherapies for BPD, the newer generalist management approaches for BPD, dismantling studies of evidence-based treatments (EBTs) for BPD, adaptations of EBTs for complex co-morbidities, and the current state of knowledge on psychopharmacologic interventions for BPD. This overview will demonstrate trends to paring down treatments to what essentially works for BPD, that most clinical settings can consider implementing as a more clear and feasible standard of care.

\section{Specialized Evidence-Based Treatments (EBTs) for BPD}

\section{Dialectical Behavioral Therapy (DBT)}

The most well-known, well researched, and widely available EBT for BPD is DBT $[39,40]$. Informed by clinical experience with suicidal personality disordered patients who did not improve with standard cognitive behavioral therapy intervention, Linehan developed DBT by incorporating the concept of dialectics and the strategy of validation into a treatment focused on skills acquisition and behavioral shaping. DBT formulates the problems of BPD as a result of the transaction between individuals born with high emotional sensitivity and "invalidating environments" that is, people or systems (i.e., families, schools, treatment settings, workplaces) that cannot perceive, understand, and respond effectively to their vulnerabilities.

DBT proposes that individuals with BPD can become more effective in managing their sensitivities and interactions with others through acquisition of skills that enhance mindfulness and enable them to better tolerate distress, regulate their emotions, and manage relationships. The full empirically validated package of DBT includes $1 \mathrm{~h}$ of weekly individual therapy, a 2-h group skills training session, out-of-session paging, and consultation team for the therapist. The intensity and structure of DBT, which is organized in an explicit, comprehensive set of manuals with instruction to therapists as well as hundreds of skills worksheets, provides an instant foundation for practitioners of any discipline or level of experience. DBT is designed for teams of clinicians and is among the most time intensive modalities for patients and clinicians. Its major mechanism of change occurs via acquisition and generalization of skills to be more emotionally regulated, mindful, and effective in the face of individual sensitivities.

\section{Mentalization-Based Treatment (MBT)}

Mentalization refers to the complex capacity human beings develop to imagine the thoughts and feelings in one's own and other's minds to understand interpersonal interactions [41]. Therein lies its mechanism of change. MBT proposes that BPD symptoms arise when a patient stops mentalizing, leading patients to operate from pathologically certainty about other's motives, the disconnection from grounding influence of reality, and a desperate need for proof of feelings through action. Attachment interactions become hyperactivated, feeding into distress and difficulty coping, rather than providing safety and security, rendering the therapeutic process with BPD difficult.

MBT aims to stabilize the problems of BPD by strengthening the patient's capacity to mentalize under the stress of attachment activation [41]. MBT therapists adopt a stance of curiosity, and "not knowing" in order to encourage patients to assess their emotional and interpersonal situation through a more grounded, flexible, and benevolent lens. Prioritizing the maintenance of mentalizing, MBT therapists support patients to think through hyperactivated states themselves, rather than providing prepackaged or intellectualized explanations, insights, or skills. Outpatient MBT involves 50 min of weekly individual therapy, $75 \mathrm{~min}$ of group therapy, and a reflecting team meeting which serves to support clinical team members in their mentalization in the process of treatment [25]. Developed within the National Health Services (NHS) in the United Kingdom, MBT provides a tenable model for treating personality disordered patients settings where patients and clinicians face scarce resources.

\section{Transference-Focused Psychotherapy (TFP)}

Based on the conceptualization of borderline personality organization introduced by Otto Kernberg in the 1960s, transference-focused psychotherapy (TFP) is a manualized, psychoanalytically oriented psychotherapy. Kernberg defined identity diffusion, primitive defense mechanisms (e.g., splitting), unstable reality testing, internally and externally expressed aggression, and conflicted internal working models of relationships as key features of personality disorders at borderline level of organization [42].

TFP's focus is the problematic interpersonal dynamics in the patient's life and their resultant intense emotional states. The patient's inherent interpersonal dynamic emerge in interactions with the therapist in the transference, and are jointly examined to resolve the splits between good and bad that drive instabilities in affect and relationships. Like MBT, TFP's 
mechanism of change occurs through helping patients achieve more balanced, integrated, and coherent ways of thinking about oneself and others. TFP involves two weekly individual therapy sessions, without group therapy. Clinicians in TFP are encouraged to receive supervision. TFP is more compatible with treatments by individual clinicians, not working in teams.

\section{Schema-Focused Therapy (SFT)}

Schema-focused therapy (SFT) is an integrative cognitive therapy focused on generating structural changes to a patient's personality. In twice weekly individual therapy sessions, the clinician uses a variety of behavioral, cognitive, and experiential techniques that focus on the therapeutic relationship, daily life outside therapy, and past traumatic experiences. Unlike the more neutral stances of other therapies, SFT encourages an attachment between therapist and client, a process described as "limited re-parenting". Therapy focuses on four schema modes of BPD: detached protector, punitive parent, abandoned/abused child, and angry/impulsive child. Its mechanism of change occurs through changing negative patterns of thinking, feeling, and behaving and developing healthier alternatives to replace them so that these dysfunctional schemas no longer control a patient's life.

\section{Generalist Approaches to BPD}

\section{General Psychiatric Management (GPM)}

Given the limitations of treatment models that require significant training and significant clinic resources, there is a need to develop, test, and disseminate less intensive treatments. One of these new EBTs is general psychiatric management (GPM) $[8 \bullet$.

GPM is based on a case management model, where interventions rely on common sense and are learned easily by generalist clinicians. Inherent in the case management approach is a focus on the patient's life outside of therapy. GPM prioritizes the attainment of stable vocational functioning over romantic relationships, as well as improvement in social functioning over specific symptom improvement. Diagnostic disclosure with a discussion of the disorder's symptoms should be the first step in psychoeducation for patients and their families, followed by information about the disorder's etiology and positive prognosis. This frames a discussion of treatment frequency and duration - treatment is only provided if it is helping the patient progress based on articulated goals. GPM rarely involves more than one weekly individual appointment. The treatment is multimodal in nature and provides guidance for psychopharmacological interventions, as well as the provision of group and family therapy and coordination across providers. Its mechanism of change is to facilitate the natural course of the disorder's improvement with specific attention to promoting functioning in endeavors outside of treatment.

What may be most specific to GPM is its central focus on an interpersonal hypersensitivity model of BPD [43]. In this model, the symptoms of BPD are understood as resulting from an emotional cascade that begins with an interpersonal stressor (e.g., separation, criticism). The therapist actively hypothesizes that any emotion dysregulation, impulsive or selfharming behavior, or hospitalization has resulted from an interpersonal problem, and works with the patient to better understand his or her sensitivities and responses.

In a randomized trial of GPM versus DBT, McMain et al. [24] found that patients in both treatments showed significant and comparable improvement across a variety of clinical outcomes measures after 1 year. Results were consistent at 2-year follow-up [44]. Furthermore, this trial later demonstrated that of patients with high Axis 1 co-morbidity, those assigned to GPM had significantly lower dropout rates than their DBT counterparts [45]. Moreover, there is evidence that attending a 1-day GPM workshop improves clinicians' attitudes about treating BPD. For example, clinicians report feeling more hopeful, competent, and open to treat BPD [46]. These findings are particularly important given that BPD is a disorder for which significant stigma may introduce barriers to successful treatment. The success of treatment dissemination depends in large part on whether clinicians are willing to use treatments and feel competent to do so.

\section{Structured Clinical Management (SCM)}

Structured clinical management (SCM) was developed in the UK, similar to GPM, reflects "best general psychiatric treatment" that is feasible for use by "generalist mental health clinicians" with minimal additional training [47, p. 57]. It was developed based on "expert consensus" about what general practices work best for treating BPD, and has been tested primarily in the context of RCTs evaluating the effectiveness of MBT [25]. Compared to patients who received MBT, those who received SCM showed substantial improvements across an array of clinical outcomes. Patients receiving MBT improved somewhat more quickly and continued to show greater benefit than SCM at 18-month follow-ups. However, those who received SCM were as well at 6 months as those in the MBT group, and showed faster reductions in self-harm.

Like GPM, SCM provides a structured framework for approaching treatment for BPD (see Table 2 for comparisons). This framework is guided by a number of generalist principles and is meant to make treatment understandable and predictable for patients. There is an emphasis on sharing the borderline diagnosis with patients, psychoeducation, alliance building that is based both on contractual (e.g., goal agreement) and relational factors (e.g., trust, reliability, liking), encouragement of family involvement, limited reliance 
Table 2 Comparison of GPM and $\mathrm{SCM}$

\begin{tabular}{|c|c|c|}
\hline & GPM [8] & SCM [47] \\
\hline Focus & $\begin{array}{l}\text { - Life outside treatment } \\
\text { - Interpersonal hypersensitivity }\end{array}$ & $\begin{array}{l}\text { - Symptom reduction } \\
\text { - Social functioning }\end{array}$ \\
\hline $\begin{array}{l}\text { Clinician } \\
\text { strategies }\end{array}$ & $\begin{array}{l}\text { - Self-disclosure } \\
\text { - Psychodynamic and } \\
\text { behavioral principles } \\
\text { - Flexibility/pragmatism } \\
\text { - Relationship building } \\
\text { - Case management } \\
\text { - Psychoeducation }\end{array}$ & $\begin{array}{l}\text { - Psychodynamic principles } \\
\text { - Borrows from DBT, CBT, Rogerian therapy, } \\
\text { and MBT } \\
\text { - Case management } \\
\text { - Psychoeducation }\end{array}$ \\
\hline Individual therapy & - 1 /week PRN if working & -1/week \\
\hline Group therapy & - Encouraged & - Required problem-solving group $1.5 \mathrm{~h} / \mathrm{wk}$ \\
\hline Family involvement & $\begin{array}{l}\cdot \text { Psychoeducation } \\
\cdot \text { Multi-family groups }\end{array}$ & $\begin{array}{l}\text { - 2-session intervention } \\
\text { - Further sessions PRN }\end{array}$ \\
\hline Intersession contact & - Use sparingly & $\begin{array}{l}\text { - Use sparingly } \\
\text { - Case management PRN }\end{array}$ \\
\hline Crisis management & $\begin{array}{l}\text { - Safety planning } \\
\text { - Chain analyses }\end{array}$ & $\begin{array}{l}\text { - Support } \\
\text { - Problem solving }\end{array}$ \\
\hline Co-morbidities & $\begin{array}{c}\text { - Specific hierarchies (e.g., BPD } \\
\text { considered primary to MDD) }\end{array}$ & $\begin{array}{l}\text { - BPD primary to MDD } \\
\text { - Substance use/dependence managed with } \\
\text { adjunctive care }\end{array}$ \\
\hline Medications & - Conservative approach & $\begin{array}{l}\text { - Conservative approach } \\
\text { - Prescribe for co-morbid conditions not BPD }\end{array}$ \\
\hline
\end{tabular}

on psychopharmacological intervention, some guidance on managing co-morbid conditions, and explicit safety planning. Both GPM and SCM recommend intersession contact be used sparingly. However, SCM takes a more cautious approach, advocating for "vigorously supporting the patient on the telephone if necessary" [47, p. 69], vehemently pursuing clients who have not come to treatment, and a willingness to meet them at home or elsewhere when safety risk is elevated. This may have more to do with differences in the legal climate of the UK versus the USA than with beliefs about the utility of intersession contact. Also, SCM includes specifically articulated weekly group therapy. Group therapy is open on a rolling basis for patients and includes psychoeducation and a framework focused on problem solving.

SCM has considerable similarity to GPM in terms of training requirements, structure, and general principles. However, descriptions of therapeutic techniques employed in SCM suggest that in some respects, it may appear more similar to MBT than GPM in practice. GPM is less psychotherapeutically oriented than other evidence-based treatments for BPD [29]. The general therapeutic stance includes responsivity, appropriate self-disclosure, flexibility, and pragmatism. The nonspecific techniques and therapeutic stance employed in SCM are generally rooted in psychodynamic principles consistent with MBT. These include authenticity and openness, the adoption of a "not knowing" stance, a focus on misunderstandings in the relationship, and generation of curiosity about belief and intentions.

\section{Dismantling Studies}

Now that several evidence-based treatments for BPD have been tested, their most essential ingredients can be discerned from dismantling studies. A major advance in the last 5 years for understanding what works in BPD treatment comes from Linehan's dismantling study of DBT. DBT in its standard form involves an intensive package of weekly individual therapy, weekly two and a half hour skills training group, 1-h consultation team for the therapist, and paging for skills coaching available between sessions. Linehan and colleagues compared this full, or "standard DBT" package to DBT individual therapy (DBT-I) without DBT skills training group as well as DBT skills training group (DBT-S) without skills coaching [30•]. All packages of DBT demonstrated significant improvements in suicidality and reduction in use of crisis services. While standard DBT showed greater improvement in frequency of self-harm, anxiety, and depression than the DBTI condition, it did not show significant gains over DBT-S despite the significant difference in total hours of treatment (average $55.3 \mathrm{~h}$ in standard DBT versus $31.7 \mathrm{~h}$ in DBT-S). 
Linehan's component analysis advances an imperative to distill the specialized and resource intensive EBTs for BPD to its most basic effective parts for the broadest, most resource efficient implementation.

\section{Other Brief Cost-Effective Options}

\section{Systems Training for Emotional Predictability and Problem Solving (STEPPS)}

Designed to supplement ongoing treatments such as medication, individual therapy, and case management, systems training for emotional predictability and problem solving (STEPPS) consists of cognitive behavioral elements, skills training, and a systems component. The STEPPS program includes 20 weeks of 2-h seminar-like group sessions. Two co-facilitators lead the sessions by following detailed lesson plans that cover three main components of STEPPS. The first component, psychoeducation, focuses on reframing BPD as an "emotional intensity disorder," correcting misconceptions about BPD, and helping participants gain an awareness of the schemas that drive their behaviors. The second, emotion management skills trainings, teaches skills to better manage the effects of BPD including distancing, communicating, and problem management. The third, behavior management skills training, covers goal setting, healthy eating, sleep, and exercise habits, self-harm avoidance, and interpersonal effectiveness. Participants are responsible for course materials and homework. The skills-based group sessions are supplemented by a single 2 -h psychoeducation and skills training session for families which accounts for the systems component of STEPPS. While STEPPS is not designed to be a stand-alone treatment, a limited body of research indicates that STEPPS' manualized, supplemental group sessions may enhance concurrent BPD treatments.

More recently, a series of studies conducted by Black and colleagues [48] explored the effectiveness of STEPPS for BPD patients with co-morbid antisocial personality disorder (ASPD) in both university and correctional settings. The findings indicate that subjects with co-morbid ASPD experienced similar or greater improvement in BPD symptoms, impulsiveness, and global symptoms than their non-ASPD counterparts and that STEPPS treatment is effective. These preliminary findings suggest that STEPPS has potential for treating a uniquely difficult to treat population: offenders with comorbid BPD and ASPD.

\section{Treatments for BPD and Major Co-morbidities}

BPD's usual complex pattern of co-morbidity $[49,50]$ is another challenging factor in its clinical management. While depression is its most common co-morbidity, co-occurring with BPD in the majority of cases [50], evidence from RCTs presented here suggest it responds to specialist and generalist approaches, and tends to improve when BPD improves [51]. Other common co-morbidities such as substance use disorders (SUDS) [32], eating disorders (EDs) [33], and post-traumatic stress disorder (PTSD) [34] have been shown to lead to poorer treatment outcomes in some trials. While some evidence exists that these co-occurring disorders respond to DBT [35], MBT [52], and GPM [53], the combination of self-harm or suicidality with active substance dependence or eating disorder often makes these patients ineligible for specific treatment programs in clinical practice. The application and adaptation of some of the EBTs reviewed here have begun to elucidate direction in the simultaneous management of these challenging co-morbidities.

\section{Substance Use Disorders (SUDs)}

Two reviews of interventions for co-occurring BPD and SUDs were published in the last 5 years $[32,38]$. These reviews indicate that DBT, in standard form as well as adapted in combination with opiate agonist medication, resulted in greater improvement of SUD, than either opiate agonist medication alone or treatment by community experts [35, 54]. In addition, dynamic deconstructive psychotherapy (DDP) [55], a manualized psychodynamic treatment for BPD and alcohol use disorders aimed at integrating and verbalizing emotional experiences as well as enhancing interpersonal identity and interactions through greater differentiation of self and other, showed greater improvements in BPD and alcohol use problems than TAU [20].

\section{Eating Disorders}

A limited number of studies have suggested that some individuals with co-morbid EDs and BPD will respond to standard DBT treatment $[35,56]$. One study adapted DBT for EDs in a 3-month inpatient treatment, with improvement in both bulimia nervosa (BN) and anorexia nervosa (AN), resulting in remission rates higher than reported for standard DBT from other studies [33]. The impact of this modified form of DBT was more robust for BN than AN. MBT has been adapted to treat the combination of BPD and ED [37], but results of this trial are yet to be published.

\section{Post-Traumatic Stress Disorder (PTSD)}

Of the three complicating co-morbidities for BPD presented here, the clearest progress in treatment in the last 5 years has developed for treatment of BPD and PTSD. While BPD patients commonly have co-occurring PTSD (approximately $30 \%$ in community samples and $50 \%$ in clinical samples) [36], guidelines for treating both simultaneously did not exist until recently. Common practice prior to these studies 
assumed BPD would need attention first, and then PTSD work would follow. Standard PTSD protocols have been contraindicated in the context of active self-harm or suicidality [57]. Two different DBT research groups have published positive trials on adaptations of DBT for co-morbid PTSD, one adding exposure protocols to standard DBT $[15 \bullet, 36]$ and the other modifying standard DBT to address severe PTSD symptoms at the outset of treatment [28]. The first of these trials [15•] focused on PTSD associated with the most complex and severe forms of trauma, for example childhood sexual abuse, in a 3month residentially based treatment, reporting significant reductions in PTSD-related symptoms when compared to TAU (waitlist). This distilled adaptation to DBT focuses on exposure with anti-dissociation skills, emotional regulation regarding shame and guilt, and acceptance on the trauma and the feelings a patient has surrounding it. The second trial [36] compared standard DBT to DBT plus a DBT prolonged exposure protocol (DBT-PE), conducted in an outpatient setting over 12 months. This second smaller study demonstrated the superiority in the enhanced DBT-PE treatment in terms of reduction of PTSDrelated symptom as well as suicidality and self-injury. These two trials suggest co-morbid PTSD and BPD can be concurrently treated with DBT and exposure protocols effectively. Bohus's trial was a larger study involving 29 patients in a naturalistic treatment setting and a waitlist comparison group of 29 patients. It employed a pared down and concentrated version of DBT without a need to prioritize attention to self-harming behaviors, or to wait for a reduction in suicidality or self-harm to start the treatment. Harned's trial was small, with ten of 17 DBT-PE patients completing the treatment, compared to five of nine completing standard DBT as its comparison group. The results of these trials suggest changing notions that these two disorders cannot be treated concurrently, or that significant stabilization need to occur prior to initiating PTSD focused work. Further research is needed to replicate these results.

\section{Pharmacology}

Compared with the growing evidence base for effective psychological treatments, pharmacologic treatments for BPD remain less well-studied. To date, no medication has been approved by the FDA for BPD or proven to definitively manage its cardinal symptoms, interpersonal impairments, and functional difficulties. Clinical applicability of the available evidence is hampered by a limited number of studies, small sample sizes, non-overlapping batteries of outcome measures, brief observation periods, and exclusion of co-morbidities in a population for whom both psychiatric and medical comorbidity are the rule rather than an exception.

Studies of prescribing practices indicate little conformity with the existing humble evidence-base. BPD patients are commonly subject to psychotropic polypharmacy, more likely to receive all classes of psychotropics, except TCAs and MAOIs
[58]. After 16 years of prospective follow-up, 18.6 and $6.9 \%$ of BPD patients report taking four or more and five or more psychotropic medications, respectively [59]. In the same cohort over 14 years of follow-up, nearly one-third consistently used PRN medication despite the absence of any evidence-based protocols for prescribing PRNs for BPD [60]. The wish to be helpful and perceived "pressure to do something" for patients presenting in acute distress reporting unrelenting agitation, anxiety, and insomnia may account for the tendency to prescribe outside of evidence-based parameters.

In an attempt to offer sensible clinical roadmaps, practice guidelines have been proposed by regulatory organizations and thought leaders. The American Psychiatric Association (APA) guidelines proposed a symptom-targeted approach using specific medication classes to treat specific BPD symptom domains [61]. Their recommendations include the use of SSRIs for symptoms of affective dysregulation and impulsivity, and low-dose atypical antipsychotics for "cognitive-perceptual" symptoms such as transient stress-induced paranoia and dissociation. These guidelines have been criticized for being based on an artificial construct of the psychobiology of personality disorders, an overreliance on reconstructions of retrospective data, and the lack of adequately powered RCTs to inform their recommendations [62].

In contrast, the UK-based National Institute for Clinical Excellence (NICE) guidelines revised in 2015 conclude from its review of available RCTs that the evidence is not robust enough to inform any prescribing practices, citing the study limitations discussed above [63]. NICE recommends against using any medications for BPD or specific associated symptoms, but suggests they should be used for true co-morbidities and hesitantly allows their short-term use during acute crisis.

Subsequent meta-analyses have extended the "symptom domain" model that informed the APA guidelines to extrapolate across variable outcome measurements between individual studies. The Cochrane review meta-analysis found that although differences between drugs were not statistically different and effects were modest at best, improvement was seen in all symptom domains of BPD with psychopharmacological treatment [7]. Affective dysregulation was found to improve with haloperidol, aripiprazole, olanzapine, lamotrigine, divalproex, and topiramate, with no significant differences between these medications or classes. Behavioral dyscontrol/ impulsivity improved with aripiprazole, topiramate, and lamotrigine. Cognitive-perceptual symptoms improved with aripiprazole and olanzapine. Interestingly, no SSRIs were found to improve any domain of symptoms and no medications alleviated certain core BPD symptoms, including avoidance of abandonment, chronic feelings of emptiness, identity disturbance, and dissociation. A second meta-analysis by Ingenhoven et al. identified a larger effect size for mood stabilizers on the treatment of behavioral dyscontrol-impulsive behaviors and anger and a more pronounced effect on global functioning, especially for lamotrigine and topiramate [64]. 
Of the psychotherapeutic approaches reviewed here, only GPM and SCM incorporates specific psychopharmacological guidelines, which combine elements of NICE and APA guidelines [8•]. Principled, yet flexible approaches to prescribing attempts to strike a balance between the evidence-based perspective that medications appear at best adjunctive while also being potentially harmful and the clinical wisdom that medication may be cautiously used in a symptom-targeted manner to promote engagement in psychotherapy. Prescribers are encouraged to avoid reactive prescribing and deliver psychoeducation about suggested medications which includes appropriate uncertainty about expected benefits.

GPM offers a specific algorithm to help prescribers limit harm and maximize potential benefit from medications. In this algorithm, no medications are prescribed for patients who do not request them and are not in distress. SSRIs are prescribed for either bona fide co-morbid major depressive episode or for patients who may have mild distress and request a medication. For behavioral dyscontrol/impulsivity and anger, either a mood stabilizer or antipsychotic can be prescribed, but risks must be discussed. For those with cognitive-perceptual disturbance, low-dose antipsychotics are recommended. In keeping with NICE guidelines, GPM promotes discontinuing medications if ineffective, avoiding polypharmacy, and time-limiting use.

Existing guidelines for pharmacotherapy in BPD do not reflect consensus but rather a chorus of distinct voices with a few overlapping refrains. One shared aim is the minimization of harms by prioritizing psychotherapy whenever possible, avoiding polypharmacy, adding medications and tapering those lacking clear efficacy in a stepwise fashion, promoting patients' collaboration through psychoeducation and joint monitoring of risks and benefits, and considering the usefulness of prescribing in view of effects on the therapeutic relationship. Finally, benzodiazepines and benzodiazepine-like substances should be avoided out of concern for promoting dependence, abuse, and behavioral disinhibition.

\section{Conclusion}

Prior to 2011, a number of highly specialized psychotherapies, (e.g., DBT, MBT, TFP, and SFT) entered the clinical scene as effective EBTs for BPD. The proliferation of these intensive EBTs turned the tide of prevailing notions that BPD was an untreatable condition. However, while effective treatments became more available, the intensity of EBTs for BPD suggested that BPD could best be treated by specialists. More recently, structured generalist management approaches such as GPM and SCM, have also been proven to work for patients with BPD. A dismantling study of DBT has offered evidence that a more pared down version of DBT, with skills training group and case management is nearly as effective as standard resource intensive packages of DBT.
Now less intensive forms of effective treatment for BPD are more available, providing hope that the general standard of care for these complex patients can be improved. Some proposals for determining which patients should received generalist care versus specialized care have recommended that patients with either greater personality dysfunction (i.e., greater numbers of personality disorder diagnoses) [52] or lack of response to generalist models of care [29] be allocated to a higher more intensive EBT. In contrast to psychotherapy trials, pharmacology trials suggest medications are adjunctive at best, and best minimized except in the treatment of co-morbidities.

Lastly, the BPD treatment research has paid increasing attention to co-morbidities such as SUDs, EDs, and PTSD. While evidence is accumulating for treatment studies in this area, clear advances in adapting DBT to treatment for PTSD has been made in the last 5 years. Now, exposure protocols for treating PTSD have been incorporated into DBT to help patients with both BPD and PTSD symptoms. Further research on adaptations of treatment for patients with more complex co-morbidities is still needed.

Acknowledgements This work was generously funded by donors to the BPD challenge grant for expanding care to underserved patients.

\section{Compliance with Ethical Standards}

Conflict of Interest Dr. Lois W. Choi-Kain declares book royalties from Springer, outside of the submitted work. Ellen F. Finch, Dr. Sara R. Masland, Dr. James A. Jenkins, and Dr. Brandon T. Unruh declare that they have no conflicts of interest.

Human and Animal Rights and Informed Consent This article does not contain any studies with human or animal subjects performed by any of the authors.

Open Access This article is distributed under the terms of the Creative Commons Attribution 4.0 International License (http:// creativecommons.org/licenses/by/4.0/), which permits unrestricted use, distribution, and reproduction in any medium, provided you give appropriate credit to the original author(s) and the source, provide a link to the Creative Commons license, and indicate if changes were made.

\section{References}

Papers of Particular Interest, Published recently, Have Been Highlighted as:

- Of Importance

1. Bateman A, Fonagy P. Effectiveness of partial hospitalization in the treatment of borderline personality disorder: a randomized controlled trial. American journal of Psychiatry. 1999.

2. Giesen-Bloo J, Van Dyck R, Spinhoven P, Van Tilburg W, Dirksen C, Van Asselt T, et al. Outpatient psychotherapy for borderline personality disorder: randomized trial of schema-focused therapy 
vs transference-focused psychotherapy. Arch Gen Psychiatry. 2006;63(6):649-58.

3. Clarkin JF, Levy KN, Lenzenweger MF, Kernberg OF. Evaluating three treatments for borderline personality disorder: a multiwave study. American Journal of Psychiatry. 2007.

4. Blum N, St. John D, Pfohl B, Stuart S, McCormick B, Allen J, et al. Systems Training for Emotional Predictability and Problem Solving (STEPPS) for outpatients with borderline personality disorder: a randomized controlled trial and 1-year follow-up. Am J Psychiatr. 2008;165(4):468-78.

5. Stoffers JM, Völlm BA, Rücker G, Timmer A, Huband N, Lieb K. Psychological therapies for people with borderline personality disorder. The Cochrane Library. 2012.

6. Ingenhoven T. Pharmacotherapy for borderline patients: business as usual or by default? The Journal of clinical psychiatry. 2015;76(4): 522-3.

7. Lieb K, Völlm B, Rücker G, Timmer A, Stoffers JM. Pharmacotherapy for borderline personality disorder: Cochrane systematic review of randomised trials. Br J Psychiatry. 2010;196(1):4-12.

8. Gunderson JG, Links PS. Handbook of good psychiatric management for borderline personality disorder. American Psychiatric Pub; 2014. This is a practical handbook of how to employ a generalist model of BPD care.

9. Koons CR, Robins CJ, Tweed JL, Lynch TR, Gonzalez AM, Morse JQ, et al. Efficacy of dialectical behavior therapy in women veterans with borderline personality disorder. Behav Ther. 2001;32(2):37190 .

10. Linehan MM, Tutek DA, Heard HL, Armstrong HE. Interpersonal outcome of cognitive behavioral treatment for chronically suicidal borderline patients. Am J Psychiatry. 1994;151(12):1771-6. doi:10.1176/ajp.151.12.1771

11. Linehan MM, Armstrong HE, Suarez A, Allmon D, Heard HL. Cognitive-behavioral treatment of chronically parasuicidal borderline patients. Arch Gen Psychiatry. 1991;48(12):1060-4.

12. Carter GL, Willcox CH, Lewin TJ, Conrad AM, Bendit N. Hunter DBT project: randomized controlled trial of dialectical behaviour therapy in women with borderline personality disorder. Aust N Z J Psychiatry. 2010;44(2):162-73.

13. Priebe S, Bhatti N, Barnicot K, Bremner S, Gaglia A, Katsakou C, et al. Effectiveness and cost-effectiveness of dialectical behaviour therapy for self-harming patients with personality disorder: a pragmatic randomised controlled trial. Psychother Psychosom. 2012;81(6):356-65.

14. Steil R, Krüger A, Dyer A, Priebe K, Feldmann Jr RE, Bohus M. DBT-PTSD. Trauma und Gewalt. 2010;4(2):106-17.

15. Bohus M, Dyer AS, Priebe K, Krüger A, Kleindienst N, Schmahl $\mathrm{C}$, et al. Dialectical behaviour therapy for post-traumatic stress disorder after childhood sexual abuse in patients with and without borderline personality disorder: a randomised controlled trial. Psychother Psychosom. 2013;82(4):221-33. This paper provides evidence of a changing practice in managing comorbid PTSD and BPD.

16. Rossouw TI, Fonagy P. Mentalization-based treatment for selfharm in adolescents: a randomized controlled trial. Journal of the American Academy of Child \& Adolescent Psychiatry. 2012;51(12):1304-13. e3

17. Davidson K, Norrie J, Tyrer P, Gumley A, Tata P, Murray H, et al. The effectiveness of cognitive behavior therapy for borderline personality disorder: results from the borderline personality disorder study of cognitive therapy (BOSCOT) trial. J Personal Disord. 2006;20(5):450.

18. Weinberg I, Gunderson JG, Hennen J, Cutter Jr CJ. Manual assisted cognitive treatment for deliberate self-harm in borderline personality disorder patients. J Personal Disord. 2006;20(5):482-92.
19. Bos EH, van Wel EB, Appelo MT, Verbraak MJ. A randomized controlled trial of a Dutch version of systems training for emotional predictability and problem solving for borderline personality disorder. J Nerv Ment Dis. 2010;198(4):299-304.

20. Gregory RJ, Chlebowski S, Kang D, Remen AL, Soderberg MG, Stepkovitch J, et al. A controlled trial of psychodynamic psychotherapy for co-occurring borderline personality disorder and alcohol use disorder. Psychother Theory Res Pract Train. 2008;45(1):28.

21. Linehan MM, Comtois KA, Murray AM, Brown MZ, Gallop RJ, Heard HL, et al. Two-year randomized controlled trial and followup of dialectical behavior therapy vs therapy by experts for suicidal behaviors and borderline personality disorder. Arch Gen Psychiatry. 2006;63(7):757-66.

22. Doering S, Hörz S, Rentrop M, Fischer-Kern M, Schuster P, Benecke C, et al. Transference-focused psychotherapy v. treatment by community psychotherapists for borderline personality disorder: randomised controlled trial. Br J Psychiatry. 2010;196(5):389-95.

23. Sachdeva S, Goldman G, Mustata G, Deranja E, Gregory RJ. Naturalistic outcomes of evidence-based therapies for borderline personality disorder at a university clinic a quasi-randomized trial. J Am Psychoanal Assoc. 2013;61(3):578-84.

24. McMain SF, Links PS, Gnam WH, Guimond T, Cardish RJ, Korman $\mathrm{L}$ et al. A randomized trial of dialectical behavior therapy versus general psychiatric management for borderline personality disorder. American Journal of Psychiatry. 2009.

25. Bateman A, Fonagy P. Randomized controlled trial of outpatient mentalization-based treatment versus structured clinical management for borderline personality disorder. American Journal of Psychiatry. 2009.

26. Jørgensen CR, Freund C, Bøye R, Jordet H, Andersen D, Kjølbye $\mathrm{M}$. Outcome of mentalization-based and supportive psychotherapy in patients with borderline personality disorder: a randomized trial. Acta Psychiatr Scand. 2013;127(4):305-17.

27. Gabbard GO. Do all roads lead to Rome? New findings on borderline personality disorder. Am J Psychiatr. 2007;164(6):853-5.

28. Bateman AW, Krawitz R. Borderline personality disorder: an evidence-based guide for generalist mental health professionals. Oxford, United Kingdom: Oxford University Press; 2016.

29. Choi-Kain LW, Albert EB, Gunderson JG. Evidence-based treatments for borderline personality disorder: implementation, integration, and stepped care. Harvard Review of Psychiatry. 2016;24(5): 342-56.

30. Linehan MM, Korslund KE, Harned MS, Gallop RJ, Lungu A, Neacsiu AD, et al. Dialectical behavior therapy for high suicide risk in individuals with borderline personality disorder: a randomized clinical trial and component analysis. JAMA psychiatry. 2015;72(5):475-82. This is the first paper analyzing essential components of effective intensive treatment for BPD.

31. Farrell JM, Shaw IA, Webber MA. A schema-focused approach to group psychotherapy for outpatients with borderline personality disorder: a randomized controlled trial. J Behav Ther Exp Psychiatry. 2009;40(2):317-28.

32. Pennay A, Cameron J, Reichert T, Strickland H, Lee NK, Hall K, et al. A systematic review of interventions for co-occurring substance use disorder and borderline personality disorder. J Subst Abus Treat. 2011;41(4):363-73.

33. Kröger C, Schweiger U, Sipos V, Kliem S, Arnold R, Schunert T, et al. Dialectical behaviour therapy and an added cognitive behavioural treatment module for eating disorders in women with borderline personality disorder and anorexia nervosa or bulimia nervosa who failed to respond to previous treatments: an open trial with a 15-month follow-up. J Behav Ther Exp Psychiatry. 2010;41(4): 381-8.

34. Barnicot K, Priebe S. Post-traumatic stress disorder and the outcome of dialectical behaviour therapy for borderline personality disorder. Personal Ment Health. 2013;7(3):181-90. 
35. Harned MS, Chapman AL, Dexter-Mazza ET, Murray A, Comtois KA, Linehan MM. Treating co-occurring Axis I disorders in recurrently suicidal women with borderline personality disorder: a 2-year randomized trial of dialectical behavior therapy versus community treatment by experts. J Consult Clin Psychol. 2008;76(6):1068-75. doi:10.1037/a0014044.

36. Harned MS, Korslund KE, Linehan MM. A pilot randomized controlled trial of Dialectical Behavior Therapy with and without the Dialectical Behavior Therapy Prolonged Exposure protocol for suicidal and self-injuring women with borderline personality disorder and PTSD. Behav Res Ther. 2014;55:7-17.

37. Robinson P, Barrett B, Bateman A, Hakeem A, Hellier J, Lemonsky $F$, et al. Study protocol for a randomized controlled trial of mentalization based therapy against specialist supportive clinical management in patients with both eating disorders and symptoms of borderline personality disorder. BMC psychiatry. 2014;14(1):1.

38. Lee NK, Cameron J, Jenner L. A systematic review of interventions for co-occurring substance use and borderline personality disorders. Drug and alcohol review. 2015;34(6):663-72.

39. Linehan MM. Cognitive-behavioral treatment of borderline personality disorder: Guilford Press; 1993.

40. Linehan MM. Skills training manual for treating borderline personality disorder: Guilford Press; 1993.

41. Bateman A, Fonagy P. Mentalization-based treatment for borderline personality disorder: a practical guide. OUP Oxford; 2006.

42. Kernberg O. Borderline personality organization. J Am Psychoanal Assoc. 1967;15(3):641-85.

43. Gunderson JG, Lyons-Ruth K. BPD's interpersonal hypersensitivity phenotype: a gene-environment-developmental model. J Personal Disord. 2008;22(1):22.

44. McMain SF, Guimond T, Streiner DL, Cardish RJ, Links PS. Dialectical behavior therapy compared with general psychiatric management for borderline personality disorder: clinical outcomes and functioning over a 2-year follow-up. American Journal of Psychiatry. 2012.

45. Wnuk S, McMain S, Links PS, Habinski L, Murray J, Guimond T. Factors related to dropout from treatment in two outpatient treatments for borderline personality disorder. J Personal Disord. 2013;27(6):716

46. Keuroghlian AS, Palmer BA, Choi-Kain LW, Borba CP, Links PS, Gunderson JG. The effect of attending good psychiatric management (GPM) workshops on attitudes toward patients with borderline personality disorder. J Personal Disord. 2015;1-10

47. Bateman A, Krawitz R. Borderline personality disorder: an evidence-based guide for generalist mental health professionals. Oxford: Oxford University Press; 2016.

48. Black DW, Simsek-Duran F, Blum N, McCormick B, Allen J. Do people with borderline personality disorder complicated by antisocial personality disorder benefit from the STEPPS treatment program? Personal Ment Health. 2016;10(3):205-15. doi:10.1002 /pmh.1326.

49. Zanarini MC, Frankenburg FR, Hennen J, Reich DB, Silk KR. Axis I comorbidity in patients with borderline personality disorder: 6year follow-up and prediction of time to remission. Am J Psychiatr. 2004;161(11):2108-14.
50. Zimmerman M, Mattia JI. Axis I diagnostic comorbidity and borderline personality disorder. Compr Psychiatry. 1999;40(4):245-52.

51. Gunderson JG, Stout RL, Shea MT, Grilo CM, Markowitz JC, Morey LC, et al. Interactions of borderline personality disorder and mood disorders over 10 years. J Clin Psychiatry. 2014;75(8): 829-34. doi:10.4088/JCP.13m08972.

52. Bateman A, Fonagy P. Impact of clinical severity on outcomes of mentalisation-based treatment for borderline personality disorder. Br J Psychiatry. 2013;203(3):221-7.

53. Boritz T, Barnhart R, McMain SF. The influence of posttraumatic stress disorder on treatment outcomes of patients with borderline personality disorder. J Personal Disord. 2016;30(3):395-407.

54. Linehan MM, Dimeff LA, Reynolds SK, Comtois KA, Welch SS, Heagerty P, et al. Dialectical behavior therapy versus comprehensive validation therapy plus 12 -step for the treatment of opioid dependent women meeting criteria for borderline personality disorder. Drug Alcohol Depend. 2002;67(1):13-26.

55. Gregory RJ, Remen AL. A manual-based psychodynamic therapy for treatment-resistant borderline personality disorder. Psychotherapy: Theory, Research, Practice, Training. 2008;45(1): 15.

56. Telch CF, Agras WS, Linehan MM. Dialectical behavior therapy for binge eating disorder. J Consult Clin Psychol. 2001;69(6):1061.

57. Foa EB, Keane TM, Friedman MJ, Cohen JA. Effective treatments for PTSD: practice guidelines from the International Society for Traumatic Stress Studies. New York, NY: Guilford Press; 2009.

58. Zanarini MC, Frankenburg FR, Harned A, Fitzmaurice G. Rates of psychotropic medication use reported by borderline patients and axis II comparison subjects over 16 years of prospective followup. J Clin Psychopharmacol. 2015;35(1):63-7.

59. Zanarini MC, Frankenburg FR, Reich DB, Conkey LC, Fitzmaurice GM. Treatment rates for patients with borderline personality disorder and other personality disorders: a 16-year study. Psychiatr Serv. 2014;66(1):15-20.

60. Martinho Jr E, Fitzmaurice GM, Frankenburg FR, Zanarini MC. Pro re nata (as needed) psychotropic medication use in patients with borderline personality disorder and subjects with other personality disorders over 14 years of prospective follow-up. J Clin Psychopharmacol. 2014;34(4):499-503.

61. APA. Practice guideline for the treatment of patients with borderline personality disorder. American Psychiatric Association: American Psychiatric Pub; 2001.

62. Kendall T, Burbeck R, Bateman A. Pharmacotherapy for borderline personality disorder: NICE guideline. Br J Psychiatry. 2010;196(2): $158-9$.

63. National Institute for Clinical Excellence. Borderline personality disorder, treatment and management. The British Psychological society and the Royal College of Psychiatrists., London. 2009. https://www.nice.org.uk/guidance/cg78/chapter/1-Guidance. Accessed 09/02 2016.

64. Ingenhoven $\mathrm{T}$, Lafay $\mathrm{P}$, Rinne $\mathrm{T}$, Passchier J, Duivenvoorden $\mathrm{H}$. Effectiveness of pharmacotherapy for severe personality disorders: meta-analyses of randomized controlled trials. The Journal of clinical psychiatry. 2009;71(1):14-25. 